\title{
A case of visceral leishmaniasis found by left oblique hernia: A case report
}

\author{
GUOQIANG ZHANG, JIHUA ZHONG, TING WANG and LU ZHONG
}

\begin{abstract}
Department of Hematology, Renji Hospital, School of Medicine, Shanghai Jiaotong University, Shanghai 200127, P.R. China
\end{abstract}
Received July 22, 2019; Accepted January 14, 2020

DOI: $10.3892 /$ etm.2020.8487

\begin{abstract}
Visceral leishmaniasis (VL) is an infectious disease caused by Leishmania protozoa. Since sporadic cases of this disease are noted in non-endemic areas and are associated with a limited outbreak, the disease is easily overlooked. In addition, other illnesses exhibit similar symptoms. It is difficult for clinicians to establish an accurate diagnosis and develop effective treatments for this disease. The present study reported a case of a 25-year-old young man admitted to the hospital due to oblique hernia. The case was diagnosed as VL. The patient presented with persistent night sweats and fatigue as described in his admission history. However, the body temperature was normal. Routine examination revealed that the patient exhibited chronic hepatitis B infection, pancytopenia, hepatosplenomegaly, increased erythrocyte sedimentation rate, significant plasma cell infiltration in bone marrow aspirate and hypergammaglobulinemia. The retrospective analysis of the present case can improve the diagnostic accuracy and treatment rate of $\mathrm{VL}$ in non-epidemic areas.
\end{abstract}

\section{Introduction}

According to the World Health Organization, approximately 200,000-400,000 new cases of visceral leishmaniasis (VL) occur every year in the world, $90 \%$ of which are noted in Southeast Asia, Latin America and East Africa (1). Based on the continuous emergence of new VL cases, the Indian subcontinent, Nepal, and Bangladesh were affected by VL and had to postpone the plan to eliminate VL from 2015 until 2020 (2). Western cities of China such as Xinjiang, Gansu, Sichuan and other provinces as well as autonomous regions were endemic areas of VL (3). The abundant epidemiological data and the high incidence rate provided the clinicians with sufficient knowledge regarding the

Correspondence to: Professor Jihua Zhong, Department of Hematology, Renji Hospital, School of Medicine, Shanghai Jiaotong University, 160 Pujian Road, Pudong New Area, Shanghai 200127, P.R. China

E-mail: jhzhong28@163.com

Key words: visceral leishmaniasis, oblique hernia, pancytopenia, hepatosplenomegaly, polyclonal hyperimmunoglobulinemia, non-endemic area incidence of the disease in endemic areas, leading to a high detection rate. However, it is difficult to diagnose imported cases in non-epidemic areas due to factors, such as the population mobility and low attention to the disease (4). Moreover, clinical manifestations and disease symptoms or signs may produce additional confusion. The absence of the medical history of patients resident in epidemic areas may lead to misdiagnosis of the disease (5). Based on research statistics, the misdiagnosis rate of clinical VL can reach $23.4 \%$ (6). VL is characterized by intermittent fever, hepatosplenomegaly, pancytopenia and polyclonal hyperimmune globulinemia. These symptoms are easily confused with AIDS, aplastic anemia, and plasma cell systemic diseases $(7,8)$. The diagnosis of VL is based on the detection of Leishmania amastigotes in the human body $(3,9)$. This should be differentiated from Penicillium marneffei, Histoplasma, and Plasmodium (10). The prognosis of VL is very poor and it mainly depends on early diagnosis and targeted therapy. Sodium antimony gluconate treatment was recommended for the treatment of Leishmaniasis in China. However, certain studies have applied sodium antimony gluconate and amphotericin B in the treatment of relapse and refractory cases due to the development of clinical resistance $(11,12)$. In order to improve the diagnostic rate and curative effect of VL, the present study combined the latest domestic and overseas research progress with the clinical data derived from VL-suspected cases, including laboratory diagnosis, diagnostic methods, relapse and other refractory cases.

\section{Case report}

In November 2010, a 25-year-old man from the Jiangxi Province of China was admitted to a local hospital due to left oblique hernia. He had hepatosplenomegaly during physical examination and his abdominal magnetic resonance imaging scan indicated portal vein dilatation $(14 \mathrm{~mm})$. The blood examinations revealed severe pancytopenia and chronic HBV infection. Significant plasma cell infiltration (12.5\%) was noted following bone marrow aspiration. Therefore, multiple myeloma was suspected. On the 29th of December 2010, the patient was transferred to our clinic. The patient worked as an excavator driver in a gold mine in the Sichuan Province of China in the past two years. A year ago, he began to suffer from low fever and night sweats, accompanied with fatigue. Administration of a Chinese herbal medicine resulted in the elimination of the fever, whereas fatigue and intermittent nighttime sweat continued until his hospitalization. He did not receive any further treatment. 
The patient exhibited no past medical history, and did not receive any supplements, illicit drugs, raw meat or unpasteurized milk. His family and personal history did not include associated diseases or disorders. However, it was reported that he had frequent sexual intercourse with different female partners. In addition, he was indirectly exposed to farm animals and pets without having close contact with them, whereas his family was not engaged in agricultural works. He denied tick or flea infections, blood transfusion and the presence of osteodynia or arthralgia. He never smoked and was not an alcoholic. On physical examination the patient did not exhibit fever and appeared pale and sweaty. The cardiorespiratory examination was unremarkable. A markedly tender and enlarged liver and spleen were noted that were located 5 and $11 \mathrm{~cm}$ below the costal margins, respectively. No rashes, dryness of eyes, mouth ulcers, or mucocutaneous bleeding were noted. Several palpable lymph nodes with an approximate diameter of $1 \mathrm{~cm}$ were noted in the supraclavicular fossa and groin. No skin lesion or sedimentation was noted. Periorbital or peripheral extremity edema was not present. The patient exhibited a left oblique hernia, which appeared two months ago.

The laboratory values are presented in Table I. Urine and stool examinations were normal. The erythrocyte sedimentation rate was $120 \mathrm{~mm} / \mathrm{h}$ and the C-reactive protein was $12 \mathrm{mg} / \mathrm{dl}$. Severe pancytopenia was present with neutropenia, mild thrombopenia and normocytic normochromic anemia. In addition, the patient experienced hypoalbulinemia and hyperglobulinemia and immunoelectrophoresis demonstrated an elevation of IgG and $\operatorname{IgA}$ (Table I). X-ray imaging indicated no evidence of bone damage. Subsequently, a new bone marrow aspiration and biopsy were performed to confirm the previous diagnosis on the 27 th of December 2010. Bone marrow smears were rich in mononuclear cells. The percentage of erythrocyte series was $44 \%$ and the cells were uncoiled, with erythroblastic anisocytosis. The percentage of granulocyte series was $36.5 \%$ and it exhibited a light deviation to the left side. The percentage of lymphoplasmocyte series was $17.5 \%$, including heteromorphic plasmocytes (uni- and binuclear), lympho-plasmocytes and lymphocytes. The percentage of the megakaryocyte series was estimated to $2 \%$, with frequent thrombocytogenic megakaryocytes. Bone marrow biopsy indicated hypercellular bone marrow with infiltration of plasmocytes. At this stage, reactive plasmacytosis was highly suspected, although it was previously shown that certain viruses, such as hepatitis viruses, rarely cause this degree of hepatosplenomegaly. The potential interactions caused by inflammatory conditions, chronic infections, autoimmune diseases, hypersensitivity states and malignancy, were also taken into account.

Parasite infectious etiology was suspected from the epidemic of schistosomiasis in the patient's hometown at the Jiangxi Province. However, serological analyses did not reveal bacterial infections with the exception of a past infection of respiratory syncytial viruses and HBV (Table II). The presence of antibodies against viruses, bacteria or parasites was not detected and multiple cultures of blood, urine and stool revealed negative results. The presence of anti-nuclear antibodies was negative and the concentration levels of the complement proteins were within the normal range. Positron Emission Tomography/Computed Tomography (PET/CT) revealed no evidence of malignancy, although substantial hepatosplenomegaly was evident in combination with dilatation of the portal vein $(14 \mathrm{~mm})$ and small levels of ascites (Fig. 1). Magnetic resonance portal venography (MRPVG) indicated dilatation of the spleen and the portal veins at a diameter of 1.6 and $1.5 \mathrm{~cm}$, respectively, whereas no evidence of canalization of collateral circulation, stenosis or arteriovenous shunt of major vessels was noted. On the 10th of January 2011, bone marrow biopsy was performed again and the smear sample indicated the presence of extracellular (Fig. 2) and intra-macrophage amastigotes (Fig. 3), which lacked an exteriorized flagellum. Detection method of worms in bone marrow smear: Bone marrow slides containing particles were prepared according to ICSH guidelines, using a Wright-Giemsa Stain kit (Baso Diagnostic Inc.), and then examined under a microscope. The marrow cellularity was observed under low power magnification (x100) to find suspicious cell or extracellular protozoa, and then confirm under the oil mirror $(\mathrm{x} 1,000)$ whether it is a Leishmania (Microscope model: Olympus CX23). The cells were morphologically assessed and the data revealed that they were possibly amastigotes of Leishmania (Leishmania) donovani. To confirm this diagnosis, blood samples were sent to the Institute of parasitic disease, at the Chinese Center for Disease Control and Prevention. The plasma Leishmania-donovani antibody was positive. Therefore, the present case was diagnosed as VL. The patient was immediately treated on the 12th of January with anti-leishmanial therapy intramuscularly, which consisted of $600 \mathrm{mg}$ sodium stibogluconate. The patient tolerated the 10-day-treatment and exhibited only a minimal arthralgia. Moreover, leukopenia and monocytosis disappeared at the beginning of February. Bone marrow biopsy was performed on the 21st of March in 2011 and was negative for amastigotes. The patient was discharged on the 25th of March 2011 with no medication. No relapse has been reported to date.

\section{Discussion}

VL, which is caused by the Leishmania donovani group of protozoa, is highly prevalent in mainland China. However, since the end of the $1950 \mathrm{~s}$, a national campaign was conducted, which resulted in the significant reduction of the disease epidemic (13). Sporadic cases have occurred in the newly reclaimed desert areas, such as the Xinjiang, Gansu, Sichuan, Shaanxi and Shanxi provinces (14). Sporadic cases have also occurred in Inner Mongolia and the Xinjiang Uygur Autonomous Region (14). However, the disease was still prevalent or sporadic in some cities in western China after the 1990s (4). In the present case report, a patient was included who lived during the recent two years in the Sichuan province. This region is located in the southwest of China. The north area of the Sichuan province is known as an endemic area of VL. It was speculated that he was infected there.

The immune response during the chronic infection of Leishmania is complicated. In the present case, the polyclonal hyperimmunoglobulinemia, which led to the diagnosis of reactive plasmocytosis, reflected the overstimulated state of humoral immunity. However, the immunoglobins stimulated by the parasite antigen were not primarily important for the protection of the patient and were involved in the pathogenesis of tissue lesions (15). The assessment of cytoimmunity was assessed by lymph node biopsy, which demonstrated a diffuse reactive proliferation of $\mathrm{T}$ cells. 
Table I. Laboratory values.

\begin{tabular}{|c|c|}
\hline Examination & Value \\
\hline \multicolumn{2}{|l|}{ Urinalysis } \\
\hline Glucose & $(-)$ \\
\hline Protein & $(-)$ \\
\hline Erythrocytes $(\mu 1)$ & 2 \\
\hline Leucocytes $(\mu \mathrm{l})$ & 3 \\
\hline Cast (/HP) & 0 \\
\hline Bacteria (/HP) & 0 \\
\hline \multicolumn{2}{|l|}{ Stool } \\
\hline Occult blood & $(-)$ \\
\hline Ova & $(-)$ \\
\hline \multicolumn{2}{|l|}{ Hematological values } \\
\hline $\operatorname{ESR}(\mathrm{mm} / \mathrm{h})$ & 120 \\
\hline Leucocyte $(/ \mu 1)$ & 1,300 \\
\hline \multicolumn{2}{|l|}{ Differential count $(/ \mu 1)$} \\
\hline Neutrophils $(/ \mu 1)$ & 600 \\
\hline Lymphocytes $(/ \mu 1)$ & 500 \\
\hline Monocytes $(/ \mu \mathrm{l})$ & 200 \\
\hline Eosinophils $(/ \mu 1)$ & 0 \\
\hline Erythrocyte $\left(10^{4} / \mu 1\right)$ & 272 \\
\hline Hemoglobin $(\mathrm{g} / \mathrm{ml})$ & 7.4 \\
\hline Hematocrit (\%) & 23.4 \\
\hline Platelets $\left(10^{4} / \mu 1\right)$ & 8.5 \\
\hline Anti-platelet IgG (ng/10 $\mathrm{PA}$ ) & 352.1 \\
\hline Anti-platelet IgM & 50.3 \\
\hline Anti-platelet IgA & 1.8 \\
\hline $\mathrm{CRP}(\mathrm{mg} / \mathrm{l})$ & 12 \\
\hline \multicolumn{2}{|l|}{ Blood chemical values } \\
\hline Total protein $(\mathrm{g} / \mathrm{dl})$ & 84.3 \\
\hline Albumin $(\mathrm{g} / \mathrm{dl})$ & 22.7 \\
\hline Globulin (g/dl) & 61.6 \\
\hline $\operatorname{IgG}(\mathrm{g} / \mathrm{l})$ & 39.20 \\
\hline $\operatorname{IgA}(g / l)$ & 0.52 \\
\hline $\operatorname{IgM}(\mathrm{g} / \mathrm{l})$ & 1.97 \\
\hline$\kappa(\mathrm{g} / \mathrm{l})$ & 10.4 \\
\hline$\lambda(\mathrm{g} / \mathrm{l})$ & 4.47 \\
\hline $\mathrm{BUN}(\mathrm{mmol} / \mathrm{l})$ & 4.2 \\
\hline Creatinine (mmol/l) & 51.6 \\
\hline $\mathrm{Na}(\mathrm{mmol} / \mathrm{l})$ & 130.3 \\
\hline $\mathrm{K}(\mathrm{mmol} / \mathrm{l})$ & 4.0 \\
\hline $\mathrm{Cl}(\mathrm{mmol} / \mathrm{l})$ & 107.1 \\
\hline$\beta 2-\mathrm{MG}(\mathrm{ng} / \mathrm{ml})$ & 6,246 \\
\hline Urino $\beta 2-\mathrm{MG}(\mu \mathrm{g} / \mathrm{l})$ & 248 \\
\hline $\mathrm{CD}_{3} \%$ & 23.9 \\
\hline $\mathrm{CD}_{4} \%$ & 6.7 \\
\hline $\mathrm{CD}_{8} \%$ & 6.9 \\
\hline $\mathrm{CD}_{16+56}(\mathrm{NK}, \%)$ & 1.0 \\
\hline LDH (U/l) & 187 \\
\hline AST (U/l) & 11 \\
\hline ALT (U/l) & 16 \\
\hline$\gamma-\mathrm{GT}(\mathrm{U} / \mathrm{l})$ & 32 \\
\hline
\end{tabular}

Table I. Continued.

\begin{tabular}{ll}
\hline Examination & Value \\
\hline Alkaline phosphatase (U/l) & 99 \\
Total bilirubin (mg/dl) & 20.1
\end{tabular}

(-), Negative; ESR, erythrocytes sedimentation rate; CRP, C-reaction protein; BUN, blood urea nitrogen; LDH, lactate dehydrogenase; AST, aspartate aminotransferase; ALT, alanine aminotransferase; $\gamma$-GT, $\gamma$-glutamyl transpeptidase.

Table II. Serological studies.

Tests Result

HBV-sAg, cAb, eAb

Anti-HCV Ab

Anti-CMV IgM, IgG

Anti-EBV IgM, IgG

Anti-RSV IgG

Anti-HIV Ab

Anti-HSV-1 IgG

Anti-toxoplasma IgG

Brucellosis

Anti-mycoplasma Ab

Candida Ag

Vidal's reaction

Rheumatoid factor (IU/ml)

Antistreptolysin O

15.6

Anti-dsDNA Ab

Candida Ag

Anti-nuclear Ab

CH50 (U/ml)

C3 (g/l)

45.18

$\mathrm{C} 4(\mathrm{~g} / \mathrm{l})$

0.86

0.09

$\mathrm{HBV}$, hepatitis B virus; $\mathrm{Ag}$, antigen; $\mathrm{Ab}$, antibody; $\mathrm{HCV}$, hepatitis $\mathrm{C}$ virus; CMV, cytomegalovirus; EBV, Epstein-Barr virus; RSV, respiratory syncytial viruses; HIV, human immunodeficiency virus; HSV, herpes simplex virus; Ig, immunoglobulin; CH50, complement hemolytic activity.

However, the peripheral blood examination demonstrated that the patient experienced severe leukopenia and low levels of $\mathrm{CD}_{4}, \mathrm{CD}_{8} \mathrm{~T}$ cells and NK cells. This maybe the result of a chronic stimulation of the Leishmania antigen and of a variety of general suppressive mechanisms, including soluble factors (16), sensitivity of different cell types (17) and interactions of cytokines (18).

Human infections can be asymptomatic or can manifest as oligosymptomatic and progressive diseases. The diagnosis of $\mathrm{VL}$ in an asymptomatic patient is challenging. The symptoms of $\mathrm{VL}$ consist of malaise, weakness, weight loss and high-grade intermittent fever. The liver and spleen progressively increase in size. Hyperpigmentation is also common (black fever). Peripheral 


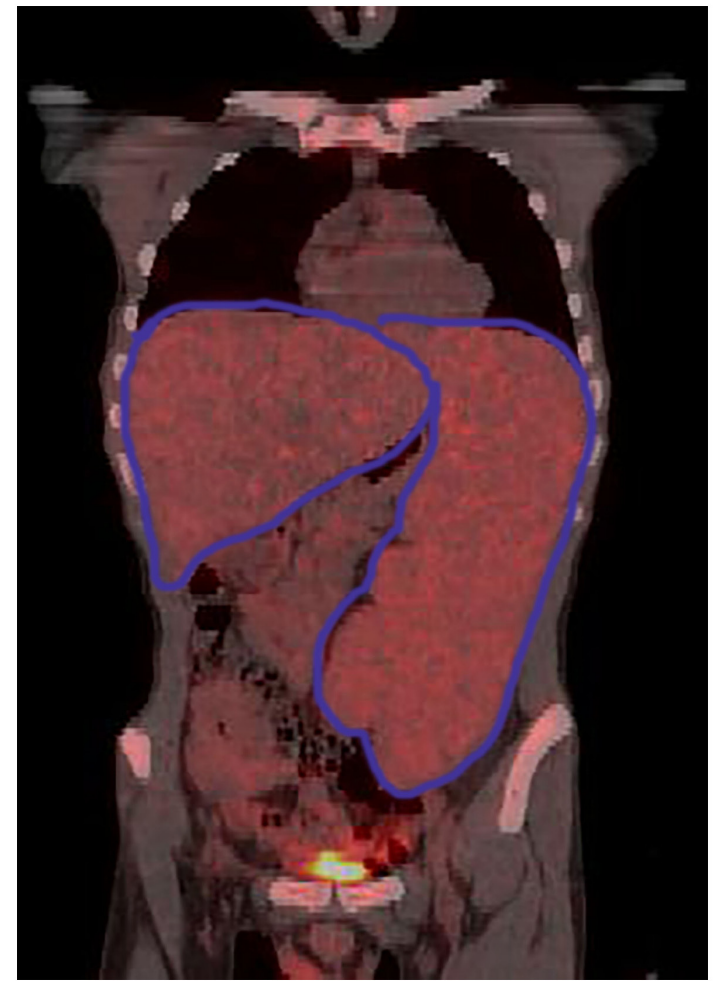

Figure 1. PET/CT indicated a significant enlarged liver and spleen (Hepatosplenomegaly with red coils in the figure). PET, Positron emission tomography; $\mathrm{CT}$, computerized tomography.

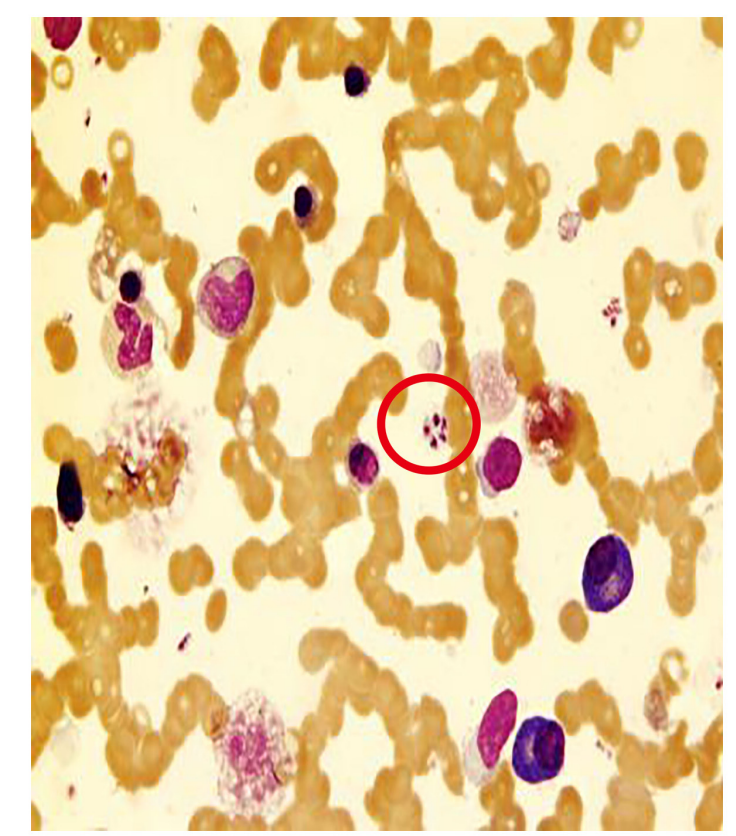

Figure 2. Bone marrow aspirate indicating extra-cellular promastigote Leishmania bodies, inside the red circle (Wright-Giemsa stain; original magnification, $\mathrm{x} 1,000)$.

blood exhibits progressive leukopenia with relative and absolute monopenia, normocytic anemia and thrombocytopenia that are encountered in the initial and late stages, respectively (19). These symptoms and the incidence of AIDS and aplastic anemia are similar with the exception that the latter two diseases are generally not associated with progressive hepatosplenomegaly.

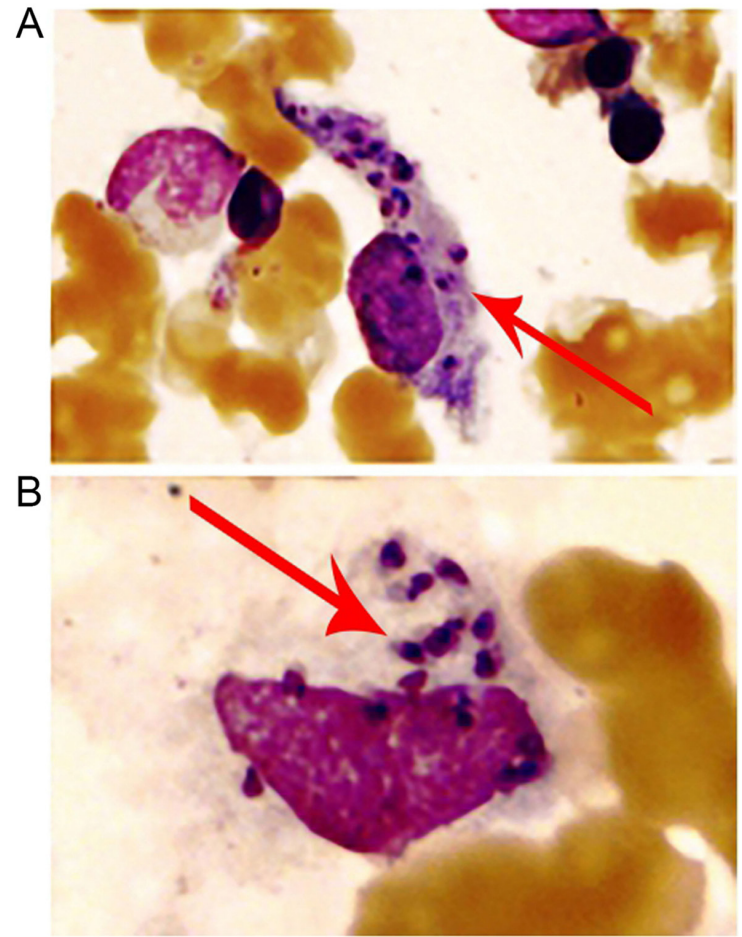

Figure 3. (A) and (B) Intracellular amastigotes (arrow pointing to the cell, Wright-Giemsa stain; original magnification, $\mathrm{x} 1,000)$.

In the present case report, the patient did not exhibit fever during his admission in our clinic and following the administration of Chinese herbal drugs. It remains unclear whether the patient's left oblique hernia was caused by a prolonged infection with Leishmania that enlarged the liver and spleen and increased abdominal pressure, or whether other factors were present that caused abnormal changes in the peritoneum. The bone marrow biopsy presented as reactive plasmacytosis and this might have led the physician to misdiagnosis. To avoid misdiagnosis of leishmaniasis with malignant B cell diseases, it is important to be aware of the differences between reactive plasmacytosis and multiple myeloma that are both present in the clinical features and pathological characteristics of these two conditions. The immunohistochemical study revealed a polytypic nature of the plasma cells. Moreover, electrophoresis was mainly indicative of a broad-based polyclonal hyperimmunoglobulinemia in reactive plasmacytosis.

The diagnosis of VL is mainly focused on the detection of Leishmania malformans in human tissues. The sensitivity of detection depends on the type of tissue. Although, the spleen aspirate is higher than $90 \%$, the risk retrieving the sample from the spleen is considerably high. The positive rate of detection is estimated from 50 to $80 \%$ in the lymph node and in blood samples (20). Leishmania no-flagellate should be readily distinguished from Penicillium marneffei, histoplasma and Plasmodium. Penicillium marneffei is a fungus and its cells are sausage-shaped, with a transverse septum. In the present study, the cell wall was detected by periodic acid-Schiff staining and the cytoplasm was not colored. The histoplasma was a fungus, which exhibited a round or oval shape. It resembled a capsule and the periodic acid-Schiff stained cell wall was red and clear. The cytoplasm was not stained. The Plasmodium was stained red-nuclear following Wright's staining and the cytoplasm was 
azure to dark blue. The malaria pigment has been shown to be brownish yellow and brown or dark brown (4).

The detection of protozoa may yield certain false negative results and it is complemented by clinical detection with serological antibody detection technology (rk39 kit). Although this method is fast and sensitive, it is used in patients with immunodeficiency with very low detection rate. Moreover, this method lacks the ability to distinguish between active or previous infections (21). Recent advances in molecular biology techniques and second-generation sequencing can improve the current method and increase the diagnostic value and dynamic monitoring of VL diseases (1).

Currently, the first-line drug used for VL in China is mainly sodium antimony gluconate. However, in other countries this treatment is combined with amphotericin B (3).

\section{Acknowledgements}

Not applicable.

\section{Funding}

No funding was received.

\section{Availability of data and materials}

All data generated or analyzed during this study are included in this published article.

\section{Authors' contributions}

GZ was mainly responsible for writing the article and data collation. JZ provided valuable assistance and information regarding the identification of cell morphology. TW was mainly responsible for the literature search work and the diagnosis of this case and the collection of differential diagnostic data. LZ directed clinical treatment and efficacy evaluation. All authors read and approved the final manuscript.

\section{Ethics approval and consent to participate}

The case has been approved by the Ethics Committee of Renji Hospital Affiliated to Shanghai Jiaotong University School of Medicine [approval no. (2019) 023]. The patient also provided written informed consent for this article.

\section{Patient consent for publication}

The patient included in the current study consented to the publication of this manuscript.

\section{Competing interests}

The authors declare that they have no competing interests.

\section{References}

1. Sundar S and Om PS: Molecular diagnosis of visceral leishmaniasis. Mol Diagn Ther 22: 443-457, 2018.
2. Medley GF, Hollingsworth TD, Olliaro PL and Adams ER: Health-seeking behaviour, diagnostics and transmission dynamics in the control of visceral leishmaniasis in the Indian subcontinent. Nature 528: S102-S108, 2015.

3. Zheng CJ, Wang LY, Xu X, Zhu XH and Wu WP: Visceral leishmaniasis in China during 2004-2007. Zhongguo Ji Sheng Chong Xue Yu Ji Sheng Chong Bing Za Zhi 27: 344-347, 2009 (In Chinese).

4. Wang JY, Cui G, Chen HT, Zhou XN, Gao CH and Yang YT: Current epidemiological profile and features of visceral leishmaniasis in people's republic of China. Parasit Vectors 5: 31, 2012.

5. Zhang WW, Ghosh AK, Mohamath R, Whittle J, Picone A, Lypaczewski P, Ndao M, Howard RF, Das P, Reed SG and Matlashewski G: Development of a sandwich ELISA to detect Leishmania 40S ribosomal protein S12 antigen from blood samples of visceral leishmaniasis patients. BMC Infect Dis 18: 500, 2018.

6. Gao Q, Liu YB, Zhong CJ and Lv XJ: Clinical analysis of 137 patients with visceral leishmaniasis. Zhongguo Ji Sheng Chong Xue Yu Ji Sheng Chong Bing Za Zhi 31: 135-157, 2013 (In Chinese).

7. Sayyahfar S, Ansari S, Mohebali M and Behnam B: Visceral leishmaniasis without fever in an 11-month-old infant: A rare clinical feature of Kala-azar. Korean J Parasitol 52: 189-191, 2014.

8. Tatarelli P, Fornaro G, Del Bono V, Nicolini LA, Raiola AM, Gualandi F, Varaldo R, Di Muccio T, Gramiccia M, Gradoni L, et al: Visceral leishmaniasis in hematopoietic cell transplantation: Case report and review of the literature. J Infect Chemother 24: 990-994, 2018.

9. Kiros YK and Regassa BF: The role of rk39 serologic test in the diagnosis of visceral leishmaniasis in a Tertiary Hospital, Northern Ethiopia. BMC Res Notes 10: 169, 2017.

10. Gui XE and Guan LR: Differential diagnosis of visceral leishmaniasis, progressive disseminated histoplasmosis and penicilliosis marneffei. Zhongguo Ji Sheng Chong Xue Yu Ji Sheng Chong Bing Za Zhi 25: 69-72, 2007 (In Chinese).

11. Editorial Board of Chinese Journal of Infectious Diseases. China expert consensus on the diagnosis and treatment of Leishmania donovani isolates from infection. Chinese Journal of Infectious Diseases 35: 513-518, 2017.

12. Hasnain MG, Nath P, Maruf S, Nabi SG, Hossain AF, Ahmed BN, Mondal D and Basher A: Amphotericin B deoxycholate for relapse visceral leishmaniasis in Bangladesh: A cross-sectional study. BMC Res Notes 11: 918, 2018.

13. Fu Q, Li SZ, Wu WP, Hou YY, Zhang S, Feng Y, Zhang LP and Tang LH: Endemic characteristics of infantile visceral leishmaniasis in the People's Republic of China. Parasit Vectors 6: 143, 2013.

14. Guan LR: Present situation of visceral leishmaniasis and prospect for its control in China. Zhongguo Ji Sheng Chong Xue Yu Ji Sheng Chong Bing Za Zhi 27: 394-397, 2009 (In Chinese).

15. Miles SA, Conrad SM, Alves RG, Jeronimo SM and Mosser DM: A role for IgG immune complexes during infection with the intracellular pathogen Leishmania. J Exp Med 201: 747-754, 2005.

16. Barral A, Carvalho EM, Badaró R and Barral-Netto $\mathrm{M}$ : Suppression of lymphocyte proliferative responses by sera from patients with American visceral leishmaniasis. Am J Trop Med Hyg 35: 735-742, 1986.

17. Carvalho EM, Bacellar O, Barral A, Badaro R and Johnson WD Jr: Antigen-specific immunosuppression in visceral leishmaniasis is cell mediated. J Clin Invest 83: 860-864, 1989.

18. Bacellar O, Brodskyn C, Guerreiro J, Barral-Netto M, Costa CH, Coffman RL, Johnson WD and Carvalho EM: Interleukin-12 restores interferon-gamma production and cytotoxic responses in visceral leishmaniasis. J Infect Dis 173: 1515-1518, 1996.

19. Carranza-Tamayo CO, Carvalho Mdo S, Bredt A, Bofil MI, Rodrigues RM, Silva AD, Cortez SM and Romero GA: Autochthonous visceral leishmaniasis in Brasília, Federal District, Brazil. Rev Soc Bras Med Trop 43: 396-399, 2010.

20. Burza S, Croft SL and Boelaert M: Leishmaniasis. Lancet 392: 951-970, 2018.

21. Antinori S, Calattini S, Longhi E, Bestetti G, Piolini R, Magni C, Orlando G, Gramiccia M, Acquaviva V, Foschi A, et al: Clinical use of polymerase chain reaction performed on peripheral blood and bone marrow samples for the diagnosis and monitoring of visceral leishmaniasis in HIV-infected and HIV-uninfected patients: A single-center, 8-year experience in Italy and review of the literature. Clin Infect Dis 44: 1602-1610, 2007. 